\title{
13-Hydroxyoctadecadienoic Acid
}

National Cancer Institute

\section{Source}

National Cancer Institute. 13-Hydroxyoctadecadienoic Acid. NCI Thesaurus. Code C131741.

A monohydroxy fatty acid resulting from the oxidation of linoleic acid or 13-hydroperoxy-

9,11-octadecadienoic acid (13-HpODE). 13-HODE may mediate physiological and pathological responses and is a potential biomarker of various human diseases, and could contribute to the progression of certain diseases. 